\title{
A Randomized Switched-Mode Voltage Regulation System for IoT Edge Devices to Defend Against Power Analysis based Side Channel Attacks
}

This paper was downloaded from TechRxiv (https://www.techrxiv.org).

\section{LICENSE}

CC BY-NC-SA 4.0

SUBMISSION DATE / POSTED DATE

24-08-2021 / 27-08-2021

\section{CITATION}

Khan, Mohd; Chen, Yu (2021): A Randomized Switched-Mode Voltage Regulation System for loT Edge Devices to Defend Against Power Analysis based Side Channel Attacks. TechRxiv. Preprint. https://doi.org/10.36227/techrxiv.16430886.v1

$\mathrm{DOI}$ 


\title{
A Randomized Switched-Mode Voltage Regulation System for IoT Edge Devices to Defend Against Power Analysis based Side Channel Attacks
}

\author{
Mohd Khan, Yu Chen \\ Department of Electrical and Computer Engineering, Binghamton University, Binghamton, NY 13902, USA \\ \{mkhan123, ychen\}@binghamton.edu
}

\begin{abstract}
The prevalence of Internet of Things (IoT) allows heterogeneous and lightweight smart devices to collaboratively provide services with or without human intervention. With an ever-increasing presence of IoT-based smart applications and their ubiquitous visibility from the Internet, user data generated by highly connected smart IoT devices also incur more concerns on security and privacy. While a lot of efforts are reported to develop lightweight information assurance approaches that are affordable to resource-constrained IoT devices, there is not sufficient attention paid from the aspect of security solutions against hardware-oriented attacks, i.e. side channel attacks. In this paper, a COTS (commercial off-the-shelf) based Randomized Switched-Mode Voltage Regulation System (RSMVRS) is proposed to prevent power analysis based side channel attacks (P-SCA) on bare metal IoT edge device. The RSMVRS is implemented to direct power to IoT edge devices. The power is supplied to the target device by randomly activating power stages with random time delays. Therefore, the cryptography algorithm executing on the IoT device will not correlate to a predictable power profile, if an adversary performs a SCA by measuring the power traces. The RSMVRS leverages COTS components and experimental study has verified the correctness and effectiveness of the proposed solution.
\end{abstract}

Index Terms-Internet of Things, Power Analysis, Side Channel Attacks, Voltage Regulator, AES, TRNG, Security.

\section{INTRODUCTION}

The prevalence of Internet of Things (IoT) allows heterogeneous and lightweight smart devices to collaboratively provide services with or without human intervention [31]. IoT architecture is being adopted by government, military, and industry. For example, Smart Factories, Smart Buildings, Smart Greenhouses and Smart Cities, etc. The IoT devices deployed at the edge networks are typically low-cost embedded systems with network capability that has a wide range of applications. Its assimilation in our daily lives is becoming increasingly common, such as smart thermostats and smart traffic light, etc. The number of IoT edge devices connected to the Internet is expected to be over 25 billion by 2030 [24].

With an ever-increasing presence of IoT-based smart applications and their ubiquitous visibility from the Internet, business transactions and user data generated by highly connected smart IoT devices also incur more concerns on security and privacy [3], [11], [25]. While a lot of efforts are reported to develop customized information assurance approaches like access control [29], [30] and lightweight blockchain [28] that are affordable to resource-constrained IoT devices, there is not sufficient attentions paid on security solutions against hardware level attacks, i.e. side channel attacks (SCA) [21]. Fortunately,the community has recognized the potential of certain security frameworks like Trusted Computing (TC) tying key platform operations to tamper-resistant on-board hardware modules (so-called TPMs) [32].

In applications where the overhead caused by the Operating System (OS) is not affordable, a bare metal solution is adopted. Moreover, some smart sensors do not require the multi-tasking benefits of OSes, so a bare metal solution is ideal. Bare metal solutions may provide optimum performance depending on the application. However, the execution of single threaded instructions on the Central Processing Unit (CPU) will reveal the power profile of the type of algorithm being executed. It opens a door to attackers, especially when a cryptography operation is executed.

A lot of research has shown that it is possible to deduce the keys of a cryptography operation by carefully measuring and analyzing the power profile of the execution platform such as a CPU [8], [10], [14], [15]. For instance, when executing an AES-128 operation, it is possible to use advanced statistical methods to analyze power traces from the CPU and deduce the correct round keys. When mounting a Power Analysis SCA (P-SCA), the adversary creates a list of all possible secret keys, also known as key guesses. The statistical methods associate a confidence level to every secret key guess, and the most correct key will show a high confidence level against the corresponding key guess as shown by Fig. 1 .

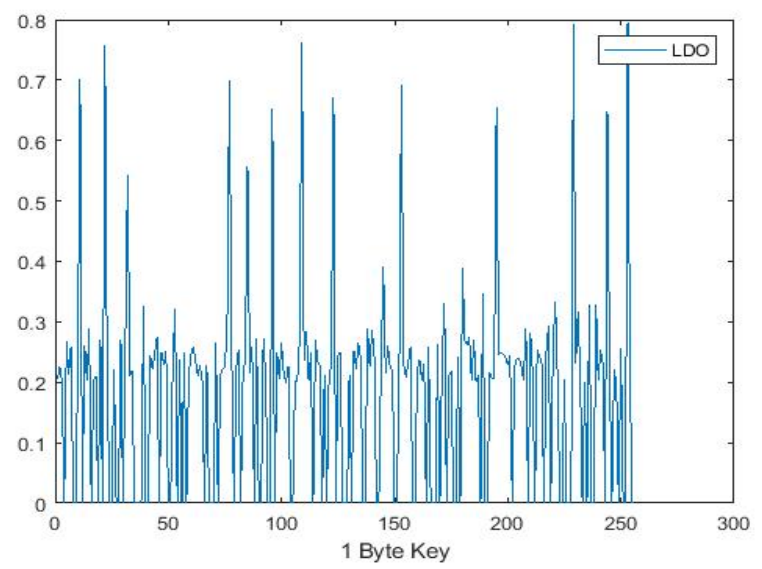

Fig. 1. Illustration of inferring keys from mounting a P-SCA. 
In this paper, a Randomized Switched-Mode Voltage Regulation System (RSMVRS) is proposed to prevent P-SCA on bare metal IoT edge devices. The RSMVRS leverages COTS (commercial off-the-shelf) devices and is implemented to direct power to IoT edge devices. The power is supplied to the target device by randomly activating power stages with random time delays. Therefore, the cryptography algorithm executing on the IoT device will not correlate to a predictable power profile if an adversary performs a SCA by measuring the power traces. The RSMVRS utilizes a NIST (National Institute of Standards and Technology)-compliant True Random Number Generator (TRNG), which will make P-SCA challenging for anyone familiar with the RSMVRS design. Thus, the proposed design is also resistant to insider attacks.

The remainder of this paper is organized as follows: Section II briefly discusses the related state-of-the-art work. Section III introduces the rationale and architecture of RSMVRS. Section IV presents the implementation of the test bench along with experiment results. Section V concludes the paper with the future work.

\section{State of The ART AND Related Work}

Side channel attacks (SCA) have been introduced to attack encryption algorithms and steal the security keys by analyzing the energy or frequency fluctuations on embedded devices [13], [23]. SCA is based on encryption algorithms that can be roughly divided into two categories: common key system based and public key based [2].

Some existing methods that are used to mitigate power analysis based SCA use (a) a capacitor in parallel to the target device, (b) an inductor in series with the target device, (c) LC filter, (d) Zener diode, and (e) another target device in parallel [10]. These methods have proven to work under normal conditions, where simply attaching a probe to the power bus will not easily reveal the power profile of the Device Under Attack (DUA). However, these methods fall short when more advanced analysis is used to perform PSCA. The passive circuit components can be easily characterized using machine learning, and the correlation between power consumption and cryptographic algorithm execution can be detected. The power traces can be further analyzed for inferring the cryptographic keys, thus making the target device vulnerable.

Moreover, there is also a decent amount of research proposing power distribution architectures to mitigate $\mathrm{P}$ SCA, such as (a) Converter Reshuffling (CoRe), (b) Timedelayed CoRe, and (c) Charge-withold CoRe [26]. The proposed architectures are addressing the P-SCA issue at the VLSI design level. However, the architectures are designed around the output of Pseudo Random Number Generator(s) (PRNG(s)), and PRNGs have been proven to be vulnerable to cryptanalysis attacks [9], [12]. The proposed RSMVRS architectures utilizes a NIST-compliant TRNG, which output random numbers in a uniform distribution and is resistant to cryptanalysis attacks.

Furthermore, in recent years there has been a lot of research conducted in designing power distribution solutions

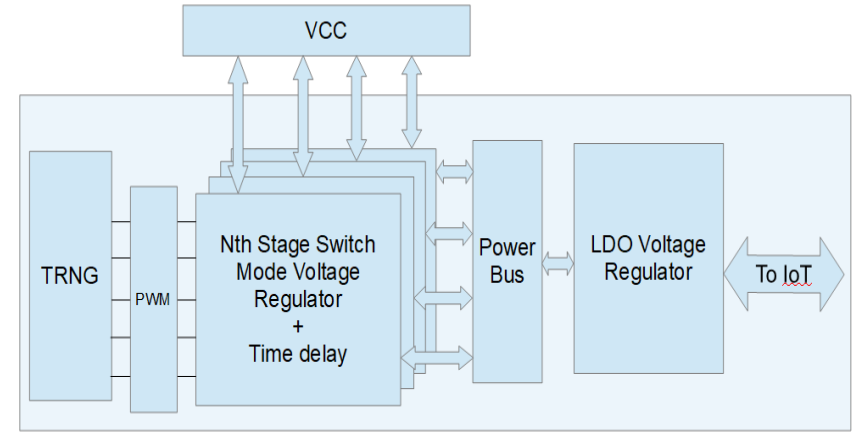

Fig. 2. RSMVRS System Overview.

for addressing the SCA vulnerability of low-power computing devices [1], [4], [5], [16], including IoT devices [6]. Most of these designs address the SCA issue at power electronics or silicon level, also keeping power efficiency in mind [18], [19]. Currently, existing work in this domain is limited in the implementations for addressing today's immediate hardware security needs of IoT bare metal edge devices. The nature of these designs in addition to the time that will take for their implementation leads to an engineering problem that is readily not addressable for today's security needs. Moreover, the time to market for such power efficient SCA resistant power regulators is extremely high. With the already increasing assimilation of IoT devices it simply does not benefit IoT hardware security domain today [26].

Therefore, a COTS-based system level security is preferred to address the current hardware security needs of the bare metal IoT edge devices. The COTS-based designs allow a readily implementation to address the SCA concerns posed by the existing power distribution mechanism in IoT edge devices. The design must be implemented in a way that not only defends against external threats, but also insider attacks such as COTS manufacturer and rogue users or designers, etc. In this paper, such a COTS based power regulation system called the RSMVRS is designed to defend against SCA [19].

\section{Randomized Switched-Mode Voltage REGULATION SYSTEM}

\section{A. System Overview}

Figure 2 illustrates the proposed Randomized SwitchedMode Voltage Regulation System (RSMVRS), which consists of a TRNG, multiple power stages and a linear voltage regulator, also known as Low-Dropout (LDO) regulator. The output from the TRNG is used to trigger the Power Width Modulation (PWM) signal for activating the power stages. In addition, there is also time delay associated with activating each power stage. The time delay is also randomly determined. The entropy is caused by duty cycle, frequency and phase (time delay) of the PWM signal.

Figure 3 shows the circuit for a single power stage of the RSMVRS. The LM2575 is a switching voltage regulator that regulates voltage primarily by turning on and off the input voltage at frequency of $52 \mathrm{KHz}$ [22]. The constant on and off produces the noise at the output voltage. Note that 


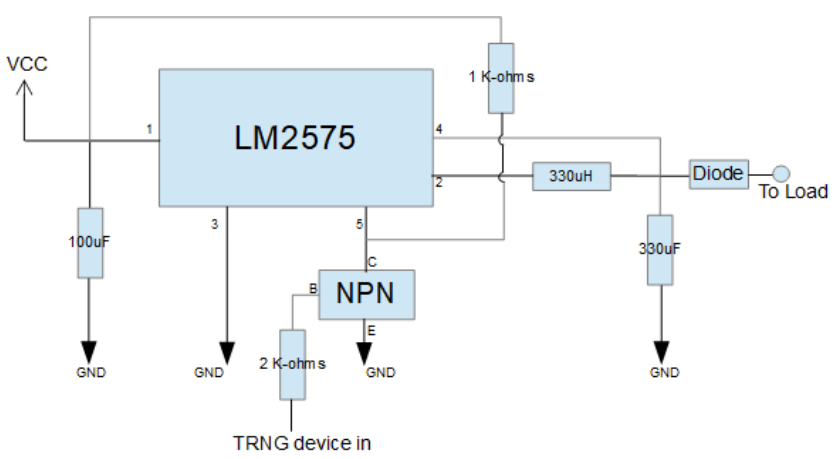

Fig. 3. Single Power Stage.

to implement RSMVRS, slight modifications are made to the LM2575's typical application circuit. First, the Zener diode is removed to prevent further regulation of voltage and contributes to the noise from the switching regulator. Second, an NPN transistor is used to control the on/off of the LM2575. The NPN transistor is triggered by a TRNG capable device.

In this study, an ATSAML22J18A is used as the TRNG device. There is a logic embedded in the ATSAML22J18A to decide when to trigger the power stages. The operation of the RSMVRS using the TRNG is shown in Fig. 4.

In this work, the activation of a particular power stage and time delay of the activation is dependent on the True Random Number (TRN). A TRN value is assigned to each power stage, and each power stage or stages are triggered when the corresponding random value is obtained from the 32-bit TRN. The time delay associated with activating a power stage is operated in a similar manner. Figure 3 can be combined in

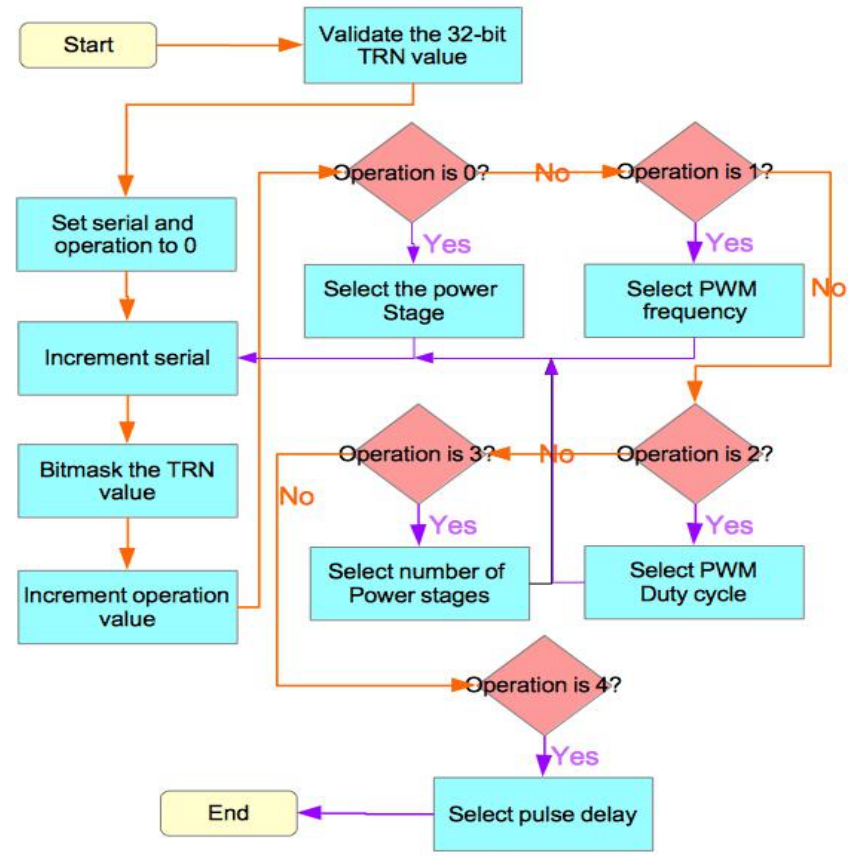

Fig. 4. RSMVRS Operation Flow.

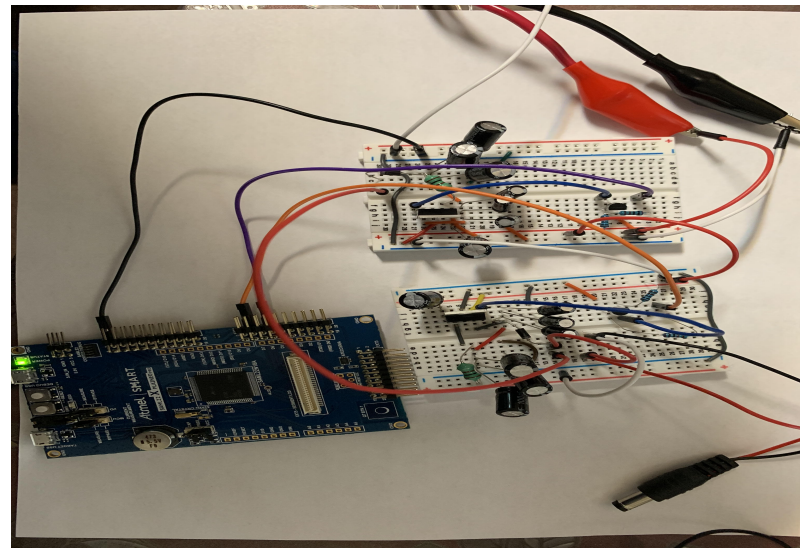

Fig. 5. RSMVRS Prototype Implementation.

any number of stages for implementing the RSMVRS.

\section{ExPERIMENTAL EVALUATION}

\section{A. Testbed Implementation}

Figure 5 illustrates the testbed implemented to evaluate the proposed RSMVRS, in which two power stages are adopted for this study. The results obtained from the circuit in Fig. 5 are discussed in detail in this section. The LM2575HV is used along with $2 \mathrm{~N} 2222$ (NPN). The GPIOs from the ATSAML22J18A Dev board are used to trigger the power stage in each breadboard.

The RSMVRS is demonstrated on an Atmel328P chipset. The Atmel328P is the DUA and operated at $4 \mathrm{MHz}$ clock in this experiment. There is no internal regulator in an Atmel328P chipset which makes it ideal to demonstrate the capability of RSMVRS. In the testbed a P-SCA is mounted on the DUA while it is executing an AES-128 operation. Power traces from the DUA are captured using a Salae Analyzer operating at $10 \mathrm{MS} / \mathrm{s}(10 \mathrm{Mhz})$. The VDD pin of the DUA is probed for capturing power traces. The Correlation Power Analysis (CPA) is used to process the captured power traces to infer the correct AES round key. The clock speed of the DUA was reduced to $4 \mathrm{MHz}$ by adjusting the clock prescaler value supported within the chipset.

Figure 6 highlights the block diagram of the experiment setup. The primary goal of the RSMVRS is to randomize the power traces while the DUA is executing the SBOX operation of the AES-128 algorithm. The multiple entropy sources embedded in the RSMVRS aid in random noise injection

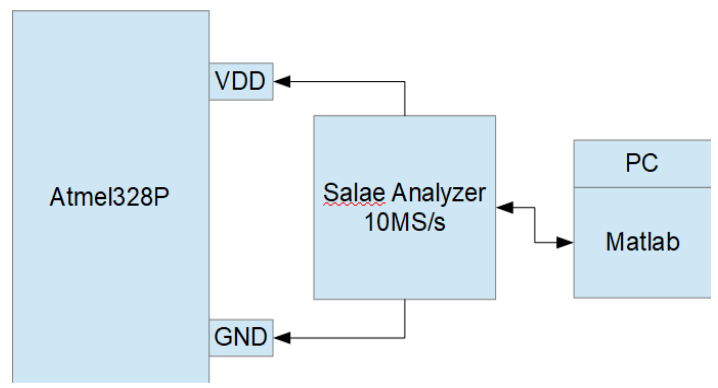

Fig. 6. Experiment Setup. 
to the power traces and thereby prevent the inference of the correct key from a CPA based attack. Moreover, the entropy sources in the RSMVRS will prevent the adversary from learning the cause-and-effect of the DUA's power profile.

A template attack is performed to extract the 16 bytes of an AES-128 round key, 1 key byte at a time. For mounting a template attack, power traces of AES-128 substitution and XORing with round key on randomly generated plaintexts are captured [27]. A large amount of power trace sample is taken to negate the effects of noise. Since the noise has a Gaussian distribution, taking a large amount of power trace samples minimized the effect of noise. Using the aforementioned method, a model is generated for every possible byte key and plaintext. This model will serve as a template for comparing the power trace from the victim's target device. A few samples are taken from the target device and a Pearsoncorrelation is performed on the captured power traces [20]. The correct round key (16 bytes) exhibits a relatively higher correlation coefficient value than other key-plaintext power models.

In a normal scenario, only an LDO regulator is used to power the DUA. The noise from the LDO regulator is normally distributed, indicated with $N$ in Eqs. (1), (2) and (3) below. Therefore, taking large samples of power traces will diffuse the effect of noise for a high SNR power model, thus allowing to infer the correct secret key as illustrated in Fig. 1. The Eq. (1) is the method used to calculate the power consumption under a normal scenario.

$$
\text { Actual Power Consumption }(\text { LDO })=(\text { Power } \pm 0.05)+
$$$$
N(\text { Environmental Noise })+N(\text { Regulator Noise })(1)
$$

For mounting a template attack, a large number of power traces from the victim's target device is taken and compared to the power model for round key matching. The noise in both the model and victim power traces is normally distributed, thereby making such an attack less tedious or time consuming for the adversary. The effects of noise on the power trace can be reversed and the cause-effect relation of power and algorithm operation can be determined, the algorithm being the AES-128's substitution operation.

The distribution of a NIST compliant TRNG is uniform, indicated with $U$ in Eq. (2) and Eq. (3) [7], [17]. The environmental and regulator noise maybe diminished by averaging large samples, but the uniform distribution of the TRNG and thereby the time delay at each power-stage will exhibit relatively higher entropy than in a normal scenario. Eq. (2) represents the noise induced by each power stage. As a result, each activated power stage will contribute to some level of entropy in the resultant power trace.

$$
\begin{aligned}
& \text { Noise at power stage } N=U(\text { TRNG }) \\
& \quad+U(\text { Time delay })+N(\text { Regulator Noise })
\end{aligned}
$$

For this experiment, a power model is generated using the power traces with higher entropy. Note that the RSMVRS will contribute to the unpredictability of power noise, and produce a less realistic power model of the target device.
The power traces from the victim's target will not have power noise with normal distribution. Therefore, the Pearsoncorrelation on the power model and power traces will not yield a useful plot for correct key combinations. The SNR of both the power model and power traces is high, more importantly, the noise effect is not easily reversed with large averaging. Eq. (3) represents the aggregate power consumption by all the power stages.

$$
\begin{gathered}
\text { Actual Power }(R S M V R S)=(\text { Power } \pm 0.05)+ \\
N(\text { EnvironmentalNoise })+\sum[\text { Noise at stage } N]
\end{gathered}
$$

Figure 1 shown earlier presents the results from a template attack. Higher correlation coefficient is observed for all the 16 bytes associated with the correct key guess. Note that this attack was mounted on a target device without the RSMVRS, therefore the current consumption of the target device is close to the current supplied by the external voltage regulator. The correlation coefficient operation minimized the noise levels. Some assumptions are made for mounting the template attack: (1) the adversary has access to the same target device; and (2) the adversary has control over the instructions being executed on the target device.

The TRNG values were obtained from a ATSAML22J18A chipset. In the plot, the wrong key guess can be noticed. The correlation coefficient operation inferred the wrong key guess due to extremely noisy power traces. Since the entropy has uniform probability density, the noise cannot be removed by taking larger samples. The random values were applied to the power model and the attack power trace as well. Since the TRNG produces random values with uniform probability density, the power model does not match the attack power trace. In other words, there is more entropy in the system with the implementation of the RSMVRS. Therefore, it will be extremely challenging for the adversary to mount a successful side channel attack. The TRNG values have uniform probability density. Therefore, the noise induced by randomly activating one or more power stages is also uniformly distributed, thus, making it challenging for the adversary to reduce the effect of noise with large sample sizes.

\section{B. Experimental Results}

Figure 7 illustrates the power trace comparison of the RSMVRS and LDO implementations. The noise in the RSMVRS is noticeable. Moreover, the average voltage is slightly higher than LDO due to power consumption by the RSMVRS circuitry. One important thing to note is that the RSMVRS is the average of 15,360 samples and LDO power samples shown is average of 10,240 data sets. The large data set has minimized the noise for LDO, but the noise levels for RSMVRS are still very pronounced. The slightly higher sample size for RSMVRS is taken to ensure that the effects of environmental noise is minimized to the fullest extent.

The distribution of the data-sets of the power traces are illustrated in Fig. 8. The RSMVRS has a mean of 5.1329 volts and standard deviation of 0.005 , whereas the LDO 


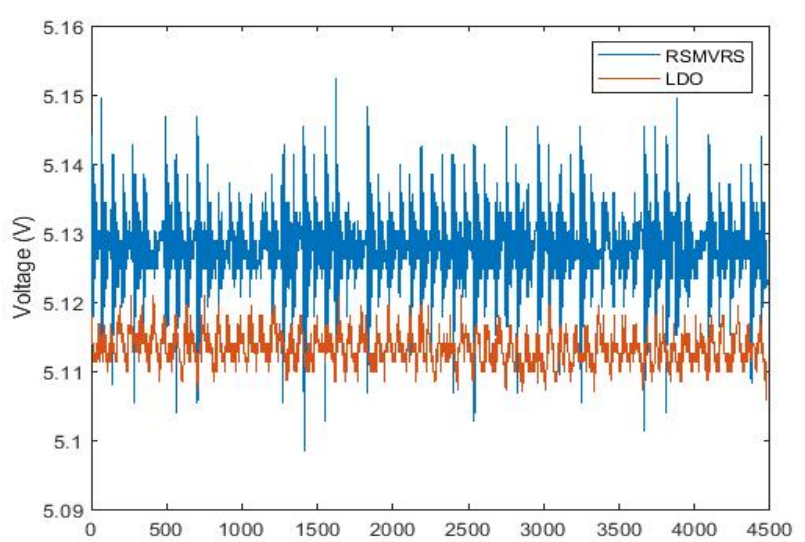

Fig. 7. Power Trace Comparison.

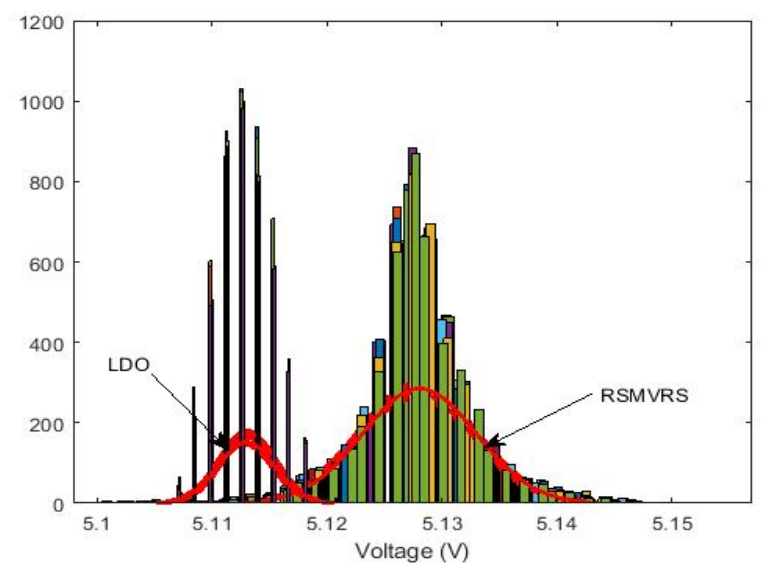

Fig. 8. Power Trace Distribution.

has a mean of 5.11304 volts and a standard deviation of 0.002. The RSMVRS demonstrated a wider spread than LDO due to the fact the entropy is higher compared to the LDO implementation. Moreover, the variation of the voltage samples for the LDO are relatively less compared to the samples for RSMVRS.

Figure 9 illustrates the results of side channel attack. The correct 16 byte round key in decimal is $32,77,85,185$, 22, 153, 96, 109, 123, 147, 253, 204, 244, 229, 195, 11. Notice in the Fig. 9 that the correct key bytes had the highest correlation coefficient for the LDO implementation. However, in the RSMVRS implementation the correlation coefficient for the same key bytes were low with the exception of a few key bytes. Only 4 out of 16 round key bytes overlap for both LDO and RSMVRS implementation.

The experimental results in Fig. 7 and Fig. 8 show that the entropy induced by each power stages of the RSMVRS system could defend against power analysis attack. It is important to note that the RSMVRS is designed to operate by activating stages in an unpredictable manner even if the working of the system is known to the adversary. And the NIST compliant TRNG plays a crucial role to make this happen.

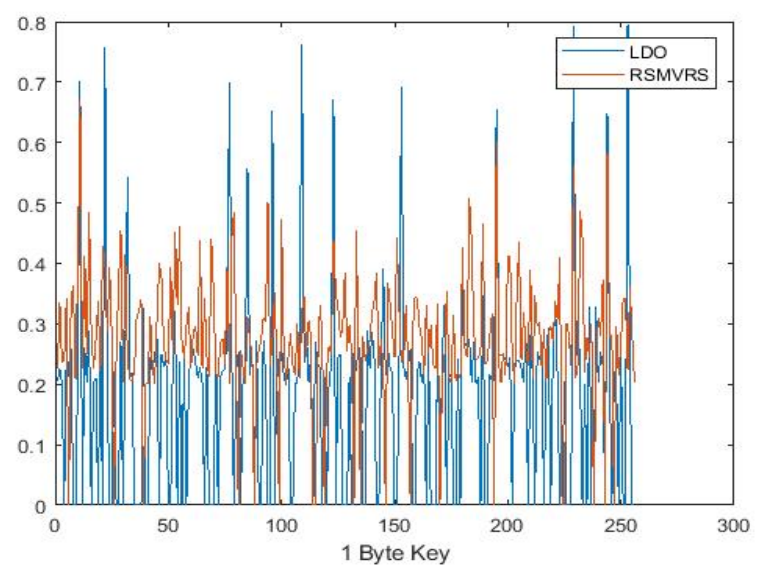

Fig. 9. Power trace comparison.

\begin{tabular}{||cccc||}
\hline & Method & \% Secret Key & \# of Samples \\
\hline \hline$[10]$ & Capacitor & $100 \%$ & 1500 \\
{$[10]$} & Inductor & $100 \%$ & 500 \\
{$[10]$} & LC Filter & $100 \%$ & 5000 \\
{$[10]$} & Zener diode & $100 \%$ & 300 \\
Proposed & RSMVRS & $25 \%$ & 10240 \\
{$[26]$} & CoRe (simulation) & Unknown & Unknown \\
\hline \multicolumn{4}{c}{ TABLE 1 }
\end{tabular}

PERFORMANCE EVALUATION

\section{Performance Evaluation}

Table I illustrates the comparison of the proposed method, RSMVRS, against other existing circuit countermeasures. For RSMVRS it took 10,240 samples sets to deduce $25 \%$ of the secret without any software countermeasures. Note that CoRe is only demonstrated in simulation, there is no result reported based on a physical implementation.

\section{Discussions and Comparison}

All the existing circuit countermeasures were evaluated against RSMVRS, which shows very promising results for mitigating P-SCA. The entire secret key could be inferred for all the existing counter measures compared to the $25 \%$ using the RSMVRS. Additionally, it took more than double the samples to infer the $25 \%$ of the secret key using RSMVRS, thus, indicating the difficulty that will be imposed to the adversary. It is important to note that these are evaluations of hardware based security measures only, and no software based countermeasures were implemented. There is a ongoing study to evaluate the effectiveness of the RSMVRS using additional software based countermeasure.

\section{Conclusions}

IN this paper, we introduced a Randomized SwitchedMode Voltage Regulation System (RSMVRS), which can be used as a mitigation for resisting side channel attacks in bare metal IoT edge devices. The experimental study has shown that in a RSMVRS implementation, the entropy induced in the power traces cannot be cancelled by taking large samples. The large samples may minimize the noise introduced due 
to external environmental factors, but the noise contributed by the intrinsic nature of the RSMVRS cannot be minimized by averaging large datasets. Moreover, it is very difficult to mount a SCA on a RSMVRS because it might be easy to probe the input and output voltage, but due to the random activation of the stages it will be challenging to perform template analysis on the power traces as demonstrated in Section IV-B. In addition to that, the random activation of power stages will also defend against insider attacks, which is crucial because of the COTS based design of the RSMVRS.

As part of our on-going efforts, the RSMVRS design can be combined with more power efficient SCA resistant voltage regulator solutions for increased protection against SCAs in a power efficient manner. In addition, with growing interest in Artificial Intelligence (AI) based SCA more research is required to determine if RSMVRS is resistant to AI-enhanced attacks.

\section{REFERENCES}

[1] G. S. W. Armin, Krieg; Johannes, "A side channel attack countermeasure using system-on-chip power profile scrambling," in IEEE 17th International On-Line Testing Symposium. IEEE, 2011, pp. 222-227.

[2] K. Chen, D. Guo, Y. Zhang et al., "A survey of side-channel attack and security assessment for cryptographic equipment," in 7th Int. Conf. on Computer Engineering and Networks, Shanghai, 2017, pp. 1-10.

[3] N. Chen and Y. Chen, "Smart city surveillance at the network edge in the era of iot: opportunities and challenges," in Smart Cities. Springer, 2018, pp. 153-176.

[4] Y. C. Z. Y. Chenguang, Wang;Ming, "Power profile equalizer: a lightweight countermeasure against side-channel attack," in IEEE 35th International Conference on Computer Design. IEEE, 2017, pp. 305312.

[5] M. B. N. G. R. S. Debayan, Das;Shovan, "Asni: Attenuated signature noise injection for low-overhead power side-channel attack immunity," in IEEE TRANSACTIONS ON CIRCUITS AND SYSTEMS. IEEE, 2018, pp. 3300-3311.

[6] M. Devi and A. Majumder, "Side-channel attack in internet of things: a survey," in Applications of Internet of Things. Springer, 2021, pp. 213-222.

[7] A. R. S. N. S. L. L. V. Dray;SanVo, "A statistical test suite for random and pseudorandom number generators for cryptographic applications," 2010.

[8] D. FRANÇOIS, DURVAUX;MARC, "SCA-Pitaya: A Practical and Affordable Side-Channel Attack Setup for Power Leakage-Based Evaluations," [Online]. Available: https://dl.acm.org/doi/pdf/10.1145/3371393, 2020.

[9] J. K. B. S. D. W. C. Hall, "Cryptanalytic attacks on pseudorandom number generators," in International Workshop on Fast Software Encryption. SpringerLink, 1998, pp. 168-188.

[10] G. Hasindu, Gamaarachchi;Harsha, "Power Analysis Based Side Channel Attack," [Online]. Available: https://arxiv.org/pdf/1801.00932.pdf, 2018.

[11] L. Horwitz, "IoT Device Security at the Edge Poses Unique Challenges," [Online]. Available: https://www.iotworldtoday.com/2021/05/07/iot-device-security-atthe-edge-poses-unique-challenges/, 2021.

[12] B. S. JChung-Chih Li, "Using linear congruential generators for cryptographic purposes."

[13] G. Joy Persial, M. Prabhu, and R. Shanmugalakshmi, "Side channel attack-survey," Int J Adva Sci Res Rev, vol. 1, no. 4, pp. 54-57, 2011.

[14] X. X. J. F. T. Jungmin, Park, "Power-based SideChannel Instruction-level Disassembler," [Online]. Available: http://jin.ece.ufl.edu/papers/DAC18.pdf, 2018.

[15] W. D. Lo, Owen;Buchanan, "Power analysis attacks on the aes-128 s-box using differential power analysis (dpa) and correlation power analysis (cpa)," in Journal of Cyber Security Technology, vol. 1. Taylor Francis Online, 2017, pp. 88-107.
[16] S. M. R. D. M. Monodeep, Kar;Arvind, "Reducing power side-channel information leakage of aes engines using fully integrated inductive voltage regulator," in IEEE JOURNAL OF SOLID-STATE CIRCUITS, vol. 53, no. 8. IEEE, 2018, pp. 2399-2414.

[17] P. Nixon, "True Random Number Generation for Cryptography," [Online]. Available: http://www.cs.tufts.edu/comp/116/archive/fall2013/pnixon.pdf.

[18] K. Orhun, Aras Uzun; Selçuk, "Converter-gating: A power efficient and secure on-chip power delivery system," in IEEE JOURNAL ON EMERGING AND SELECTED TOPICS IN CIRCUITS AND SYSTEMS. IEEE, 2014, pp. 169-172.

[19] K. Orhun, Aras Uzun;Selçuk, "Converter-gating: A power efficient and secure on-chip power delivery system," in IEEE JOURNAL ON EMERGING AND SELECTED TOPICS IN CIRCUITS AND SYSTEMS. IEEE, 2014, pp. 169-179.

[20] E. Oswald, "Power Analysis Attacks," [Presentation]. Available: http://helper.ipam.ucla.edu/publications/scws4/scws4_6576.pdf.

[21] E. Peltonen, M. Bennis, M. Capobianco, M. Debbah, A. Ding, F. GilCastiñeira, M. Jurmu, T. Karvonen, M. Kelanti, A. Kliks et al., "6g white paper on edge intelligence," arXiv preprint arXiv:2004.14850, 2020.

[22] TI, "LM2575 1-A Simple Step-Down Switching Voltage Regulator," [Online]. Available: https://www.ti.com/lit/ds/symlink/lm2575.pdf?ts=1623559676187ref_url, 2015.

[23] C. C. Tiu, "A new frequency-based side channel attack for embedded systems," Ph.D. dissertation, University of Waterloo, 2005.

[24] Y. Vardi, "Number of Internet of Things (IoT) connected devices worldwide from 2019 to 2030," [Online]. Available: https://www.statista.com/statistics/1183457/iot-connected-devicesworldwide/, 2021.

[25] — - "The Rise of Industrial IoT and How to Mitigate Risk," [Online]. Available: https://www.securityweek.com/rise-industrial-iot-andhow-mitigate-risk, 2021.

[26] Y. Weize, "Exploiting on-chip voltage regulators as a countermeasure against power analysis attacks," 2017.

[27] B.-Y. J.-Y. Won, Y.-S.;Sim, "Key schedule against template attackbased simple power analysis on a single target," Applied Sciences, vol. 3804, 2018.

[28] R. Xu and Y. Chen, "Microchain: A light hierarchical consensus protocol for iot system," arXiv preprint arXiv:1912.10357, 2019.

[29] R. Xu, Y. Chen, E. Blasch, and G. Chen, "Blendcac: A blockchainenabled decentralized capability-based access control for iots," in 2018 IEEE International Conference on Internet of Things (iThings) and IEEE Green Computing and Communications (GreenCom) and IEEE Cyber, Physical and Social Computing (CPSCom) and IEEE Smart Data (SmartData). IEEE, 2018, pp. 1027-1034.

[30] — - "A federated capability-based access control mechanism for internet of things (iots)," in Sensors and Systems for Space Applications $X I$, vol. 10641. International Society for Optics and Photonics, 2018, p. $106410 \mathrm{U}$.

[31] R. Xu, S. Y. Nikouei, D. Nagothu, A. Fitwi, and Y. Chen, "Blendsps: A blockchain-enabled decentralized smart public safety system," Smart Cities, vol. 3, no. 3, pp. 928-951, 2020.

[32] M. Ylianttila, R. Kantola, A. Gurtov, L. Mucchi, I. Oppermann, Z. Yan, T. H. Nguyen, F. Liu, T. Hewa, M. Liyanage et al., "6g white paper: Research challenges for trust, security and privacy," arXiv preprint arXiv:2004.11665, 2020. 